\title{
Associations of dietary energy density with body composition and cardiometabolic risk in children with overweight and obesity: role of energy density calculations, under-reporting energy intake and physical activity
}

\author{
Alejandro Gomez-Bruton ${ }^{1,2 *}$, Lide Arenaza $^{3}$, Maria Medrano $^{3}$, Jose Mora-Gonzalez $^{4}$, \\ Cristina Cadenas-Sanchez ${ }^{4}$, Jairo H. Migueles ${ }^{4}$, Victoria Muñoz-Hernández ${ }^{4}$, Elisa Merchan-Ramirez ${ }^{4}$, \\ Wendy Daniela Martinez-Avila ${ }^{4}$, Jose Maldonado, ${ }^{5,6}$, Maddi Oses $^{3}$, Ignacio Tobalina ${ }^{7,8}$, \\ Luis Gracia-Marco ${ }^{1,4}$, German Vicente-Rodriguez ${ }^{1,2}$, Francisco B. Ortega ${ }^{4} \dagger$ and Idoia Labayen ${ }^{3} \dagger$ \\ ${ }^{1}$ GENUD (Growth, Exercise, Nutrition and Development) Research Group, Universidad de Zaragoza, 50009 Zaragoza, Spain \\ ${ }^{2}$ Department of Physiatry and Nursing, Faculty of Health and Sport Sciences (FCSD), University of Zaragoza, 50009 Zaragoza, \\ Spain \\ ${ }^{3}$ Nutrition, Exercise and Health Research Group, Elikadura, Ariketa Fisikoa eta Osasuna, ELIKOS Group, Institute for \\ Innovation E Sustainable Development in Food Chain (IS-FOOD), Public University of Navarra, Campus de Arrosadia, 31008 \\ Pamplona, Spain \\ ${ }^{4}$ PROFITH "PROmoting FITness and Health through physical activity" Research Group, Department of Physical Education and \\ Sports Education, Faculty of Sports Science, University of Granada, 18071 Granada, Spain \\ ${ }^{5}$ Department of Pediatrics, School of Medicine, University of Granada, 18071 Granada, Spain \\ ${ }^{6}$ Institute of Biomedicine Research (Instituto de Investigación Biosanitaria (IBS), 18071 Granada, Spain \\ ${ }^{7}$ Department of Nuclear Medicine, University Hospital of Araba (HUA), 01009 Vitoria-Gasteiz, Spain \\ ${ }^{8}$ Department of Surgery Radiology and Physical Medicine, Faculty of Medicine, University of the Basque Country, UPV/EHU, \\ o1009 Vitoria-Gasteiz, Spain \\ (Submitted 30 October 2018 - Final revision received 15 January 2019 - Accepted 27 January 2019 - First published online 03 April 2019)
}

\section{Abstract}

This study examined (1) the association of dietary energy density from solid $\left(\mathrm{ED}_{\mathrm{S}}\right)$ and solid plus liquids (ED $\mathrm{SL}$ ) with adiposity and cardiometabolic risk factors (CRF) in children with overweight and obesity, (2) the effect of under-reporting on the mentioned associations and (3) whether the association between ED and body composition and CRF is influenced by levels of physical activity. In a cross-sectional design, 208 overweight and obese children (8-12-year-old; 111 boys) completed two non-consecutive $24 \mathrm{~h}$ recalls. ED was calculated using two different approaches: $\mathrm{ED}_{\mathrm{S}}$ and $\mathrm{ED}_{\mathrm{SL}}$. Under-reporters were determined with the Goldberg method. Body composition, anthropometry and fasting blood sample measurements were performed. Moderate to vigorous physical activity (MVPA) was registered with accelerometers (7-dregister). Linear regressions were performed to evaluate the association of ED with the previously mentioned variables. Neither $\mathrm{ED}_{\mathrm{S}}$ nor $\mathrm{ED}_{\mathrm{SL}}$ were associated with body composition or CRF. However, when under-reporters were excluded, ED ${ }_{\mathrm{S}}$ was positively associated with BMI ( $P=0.019)$, body fat percentage $(P=0.005)$, abdominal fat $(P=0.008)$ and fat mass index $(P=0.018)$, while ED $\mathrm{SL}_{\mathrm{L}}$ was positively associated with body fat percentage $(P=0.008)$ and fat mass index $(P=0.026)$. When stratifying the group according to physical activity recommendations, the aforementioned associations were only maintained for non-compliers. Cluster analysis showed that the low-ED and high-MVPA group presented the healthiest profile for all adiposity and CRF. These findings could partly explain inconsistencies in literature, as we found that different ED calculations entail distinct results. Physical activity levels and excluding under-reporters greatly influence the associations between ED and adiposity in children with overweight and obesity.

Key words: Nutrition: Diet: Dual-energy X-ray absorptiometry: Adiposity: Childhood: Moderate to vigorous physical activity

Childhood overweight and obesity pose a major public health concern worldwide, as they have shown to be responsible for increases in CVD risk factors ${ }^{(1)}$, psychological problems ${ }^{(2)}$ and orthopaedic problems ${ }^{(3)}$, among many other complications. Moreover, an extensive body of research indicates that children with obesity are at a higher risk of becoming adults with obesity compared to their counterparts without obesity ${ }^{(4)}$.

Abbreviations: BF\%, body fat percentage; DXA, dual-energy X-ray absorptiometry; ED, energy density; EDS, energy density from solid; EDSL, energy density from solid plus liquids; EI, energy intake; FMI, fat mass index; MVPA, moderate to vigorous physical activity.

* Corresponding author: A. Gomez-Bruton, email bruton@unizar.es

$\dagger$ Equal contribution. 
Although the aetiology of obesity is multifactorial and complex, it is clear that physical activity and dietary habits play important roles in the development of this disease as they can induce an energy imbalance and promote excessive fat deposition. Focusing on nutrition, a range of specific nutrients and foods have been suggested as important dietary determinants of obesity in childhood and adolescence, including fruit and vegetables ${ }^{(5)}$, fibre ${ }^{(6)}$ and sugar-sweetened beverages ${ }^{(7)}$. Although the individual effect of the previously mentioned dietary components is important, it might not reflect the overall effect of diet. For this purpose, dietary energy density (ED) has been suggested as an appropriate measure of overall diet, specifically when evaluating the association between diet and obesity/adiposity $^{(8)}$.

A recent systematic review aiming to evaluate the association between ED and obesity ${ }^{(8)}$ including fourteen studies with children and adolescents found inconsistent results in this population, with some studies showing an association between ED and body composition ${ }^{(9-11)}$ and others reporting no significant associations between both variables ${ }^{(12-14)}$. Surprisingly, few studies ${ }^{(15)}$ including children assessed the association between ED and cardiometabolic risk factors (i.e. blood pressure and TAG levels), which have shown to be increased in children with obesity ${ }^{(16)}$. Although most studies presented body composition as an outcome variable, some studies used BMI, while others used body fat or anthropometric variables (skinfold thickness or waist circumference) as main outcomes, which could partly explain the mentioned inconsistent results among studies.

Another factor that could explain these inconsistencies is the definition of ED, as some researchers use solids to compute it $\left(E D_{S}\right)$, while others use a combination of solids and liquids $\left(\mathrm{ED}_{\mathrm{SL}}\right)$. McCaffrey et al. ${ }^{(17)}$ used five different classifications of ED and found that the definitions that did not include beverages showed the best association with changes in fat mass. Besides the definition of ED, it is obvious that other external factors could regulate the association between ED and body composition, such as physical activity, that was not registered in several previous studies ${ }^{(9,17,18)}$, or was registered through questionnaires $^{(19-21)}$, which have shown to have a limited validity and reliability in previous studies ${ }^{(22)}$.

Two studies evaluated the association between ED and body composition in children with obesity. The first developed by Butte et $a l .{ }^{(12)}$ found that ED did not predict weight gain during 1 year follow-up, while a recent study developed by Aburto et $a l^{(23)}$ found a positive association between ED and overweight and obesity. Interestingly, the association was stronger when only plausible reporters (i.e. excluding from the analyses both under- and over-reporters) were considered. Taking into account that previous studies have found energy intake (EI) under-reporting to be more prevalent and severe among children and adolescents with a higher $\mathrm{BMI}^{(24,25)}$, identifying under-reporters when measuring the association between ED and body composition in children with overweight and obesity is of great importance.

It therefore seems timely to evaluate the association between ED and different body composition variables (i.e. BMI, adiposity) and cardiometabolic markers, taking into account levels of objectively measured physical activity, to ascertain whether ED is critical to body composition and whether physical activity modulates this association in children with overweight and obesity, a population in which EI under-reporting has been shown to be more prevalent and severe ${ }^{(24,25)}$. Consequently, the aims of the present study were (1) to evaluate the association of $\mathrm{ED}_{\mathrm{S}}$ and $\mathrm{ED}_{\mathrm{SL}}$ with adiposity and cardiometabolic risk in children with overweight and obesity, (2) to estimate the effect of under-reporting on the mentioned associations and (3) to assess whether the association between ED and body composition/cardiometabolic risk is influenced by levels of physical activity.

\section{Methods}

\section{Participants}

Participants were recruited from two different cities of Spain (Granada and Vitoria-Gasteiz), as part of two projects that are briefly explained below:

(a) The ActiveBrains project (NCT02295072) that is described in detail elsewhere ${ }^{(26)}$ was developed in the city of Granada (south Spain) and aimed to examine the effects of a physical exercise program on brain, cognition, academic achievement as well as physical and mental health in 110 overweight or obesity participants aged 8-11. Although the ActiveBrains is a randomised controlled trial, data from the first cross-sectional evaluation were used for the present study (data collected from November 2014 to February 2016 in three different waves). The study protocol was approved by the Review Committee for Research Involving Human Subjects at the University of Granada (Ref. 848).

(b) The EFIGRO project (NCT02258126), developed in Vitoria-Gasteiz (north Spain), aimed to measure 160 children with overweight or obesity aged 8-12 and determine the effects of a multidisciplinary intervention on hepatic fat fraction and cardiometabolic risk factors. Data for the first cross-sectional evaluation were used for the present study (data collected from October 2014 to January 2017 in five different waves). The study protocol was approved by the Ethic Committee of Clinical Investigation of Euskadi (PI2014045). More details of the EFIGRO project can be found in the methodological manuscript ${ }^{(27)}$.

Following the inclusion criteria of the previously mentioned projects, the main inclusion criteria for the present study were as follows: (1) children between 8 and 12 years old, (2) children classified as overweight or obese based on the sex- and agespecific international BMI standards ${ }^{(28)}$ and (3) children who were not taking medications that influenced the central nervous system function.

From all the measured participants of the ActiveBrains and the EFIGRO projects, 208 (101 from ActiveBrains and 107 from the EFIGRO project) presented complete data for body composition and accelerometry and were, therefore, included in the study. From the 208 included participants, six did not report their pubertal status, seven did not have blood samples and eighteen did not have data for dual-energy X-ray absorptiometry (DXA) abdominal regions (not collected due to technical reasons). These participants were included in the analyses and 
consequently the number of participants for each analysis varied slightly and is specified in the 'Results' section.

\section{Body composition}

Body composition assessments were performed in the morning in a non-fasted state.

Anthropometric variables. Using a wall stadiometer and electronic scale, respectively (SECA model), the height and weight were measured in children wearing minimal clothing and no shoes. BMI was calculated as weight in kilograms divided by the height in meters square, and participants were classified into BMI categories according to the World Obesity Federation cut-offs ${ }^{(28)}$. Waist circumference was measured at the narrowest point by standard procedures with an anthropometric non-elastic tape (SECA 200).

Body fat. DXA (Hologic QDR $4500 \mathrm{~W}$ ) was used to evaluate body composition. A whole-body scan was performed from which, whole-body fat percentage $(\mathrm{BF} \%)$ and fat mass index (FMI), expressed as body fat divided by height expressed in meters square were obtained. In addition, a regional analysis was performed to assess abdominal adiposity following the protocol of previous studies ${ }^{(29)}$. One region was calculated as a rectangle drawn on the digital scan image with the lower border of the rectangle at the lower horizontal border on the top of iliac crest and the upper border established parallel with the end of the lowest rib (this region was defined as R1 in previous studies $\left.{ }^{(29)}\right)$. The lateral side of the defined region was adjusted to include all the body tissues. As this region might be larger (and therefore include more fat) in taller participants, it was adjusted by height and was called abdominal FMI.

\section{Dietary intake assessment}

Total energy intake. Following the European Food Safety Authority guidelines ${ }^{(30)}$, total EI was obtained from two nonconsecutive $24 \mathrm{~h}$ recalls referring to weekdays which were collected by trained nutritionists, conducted in the presence of the child's parents or legal guardians. A photographic manual of food portion size was used to improve the estimated amount of dietary intake. All data were registered by the Easydiet software (Biocentury ${ }^{\circledR}$, S.L.U. 2016), which is the software supported by the Spanish Association of Dietetics and Nutritionists.

Dietary ED was calculated by following two different approaches.

(a) Only $\mathrm{ED}_{\mathrm{S}}$ : total EI from solid foods relative to total grams of solid foods consumed, which is expressed as $\mathrm{kJ} / \mathrm{g}$. This calculation was done based on solid foods only, excluding all energy-containing and non-energy-containing beverages (tea, coffee, water, soft drinks, fruit juice and milk).

(b) $\mathrm{ED}_{\mathrm{SL}}$ : the $\mathrm{ED}$ of food and energy-containing beverages.

Miss-reporters. The Goldberg ${ }^{(31)}$ and Black's ${ }^{(32)}$ equations (Table 1) were used to estimate the possible under- and overreporters (children who reported non-plausible EI). This method is used to predict total energy expenditure using physical activity levels and BMR which was estimated with three different equations ${ }^{(33,34)}$ : the Schofield equation ${ }^{(34)}$ and two different equations proposed by Lazzer ${ }^{(33)}$ both taking Tanner stage into account with one using body weight ( Lazzer $_{\text {weight }}$ ) and the other using fat mass and fat-free mass from DXA $\left(\right.$ Lazzer $_{\text {DXA }}$ ). The three equations are defined in Table 1. Previous validation studies demonstrated that the Goldberg equation presents a high predictive value for detecting under-reporters when compared to doubly labelled water ${ }^{(35)}$. Specific details of the formula and used CV are presented in Table 1.

\section{Cardiometabolic variables}

Blood samples were collected after an overnight fast. Glucose was analysed using the glucose oxidase method with automatic analysers (Roche-Hitachi Modular P and D Autoanalyser; Roche Laboratory Systems), and plasma insulin was analysed by RIA using automatic microparticle analysers (Axsym; Abbott Laboratories). TAG, total cholesterol LDL and HDL were measured using an automatic analyser (Roche-Hitachi Modular P and D Autoanalyser). Blood pressure was assessed with the automatic $\mathrm{OMRON}^{\oplus} \mathrm{M} 6$ device. Mean arterial blood pressure and two different cardiometabolic (CM) risk scores (following the indications of Nyström et $\left.a l^{(36)}\right)$ were calculated as described in Table 1.

\section{Physical activity}

For seven consecutive days $(24 \mathrm{~h})$, physical activity was assessed with tri-axial accelerometers (GT3x+ and wGT3X-BT; ActiGraph) attached to the non-dominant wrist. ActiGraph.csv files were analysed with R-package GGIR version 1.2 (http://cran.r-project.org) ${ }^{(37)}$. Data of participants were included in the analyses if they recorded at least one weekend day and three week valid days ( $\geq 16$ wearing hours), as previously recommended $^{(38)}$. Identification of waking and sleeping hours was done using an automatised algorithm guided by the times reported by the participants as explained by Van Hees et al. ${ }^{\text {(39) }}$. The Hildebrand et $a l .{ }^{(40)}$ cut-off points were used to classify moderate to vigorous physical activity (MVPA).

\section{Socio-demographic status}

Parental educational level was registered for its known influence of nutritional status ${ }^{(41)}$. Mothers' educational highest degree was registered, and mothers were classified as low education (compulsory secondary school), medium education (high school) and higher education (university). Pubertal development was registered through direct examination by trained paediatricians following the Tanner scale $e^{(42)}$.

\section{Statistical analysis}

Normality of the variables was checked with histograms. For non-normal variables, data were transformed to obtain normalised variables with mean 0 and standard deviation 1 . Mean 
Table 1. Calculations of cardiometabolic (CM) risk, resting metabolic rate and number of under-reporters

CM risk
CM risk 1
CM risk 2
MAP
Resting metabolic rate $(k c a l)$
Lazzer 1
Lazzer 2
Schofield boys $<10$ years
Schofield girls $<10$ years
Schofield boys $>10$ years
Schofield girls $>10$ years
Goldberg equation
Imputed values
Cut-off points

Total number of under-reporters Under-reporters Lazzer 1 Under-reporters Lazzer 2 Under-reporters Schofield

\author{
$\left(\mathrm{N} \_\right.$waist $+\mathrm{N} \_$mean blood pressure $+\mathrm{N} \_T A G+\mathrm{N} \_$glucose $+\mathrm{N} \_$inverted $\left.\mathrm{HDL}\right) / 5$ \\ (N_TAG/HDL + N_waist + N_insulin + N_MAP)/4 \\ Diastolic blood pressure $+(0.333 \times$ (systolic blood pressure - diastolic blood pressure $))$
}

$((\mathrm{FFM}(\mathrm{kg}) \times 0.082)+(\mathrm{FM}(\mathrm{kg}) \times 0.037)-($ Tanner stage $\times 0.125)+(\mathrm{sex} \times 0.706)+2.528) \times 239.006$

$((\mathrm{BW}(\mathrm{kg}) \times 0.044)+($ height $(\mathrm{m}) \times 2.836)-($ Tanner stage $\times 0.148)+(\mathrm{sex} \times 0.781)-0.551) \times 239.006$

$19.6 \times \mathrm{BW}(\mathrm{kg})+1.033 \times$ height $(\mathrm{m})+414.9$

$16.97 \times$ BW $(\mathrm{kg})+1.618 \times$ height $(\mathrm{m})+371.2$

$16.25 \times$ BW $(\mathrm{kg})+1.372 \times$ height $(\mathrm{m})+515.5$

$8.365 \times$ BW $(\mathrm{kg})+465 \times$ height $(\mathrm{m})+200$

$\mathrm{EI}_{\text {rep }} / \mathrm{BMR}>\mathrm{PAL} \times \exp \left( \pm 2 \times \frac{(S / 100)}{\sqrt{n}}\right)$ where $S=\sqrt{\frac{\mathrm{CV}_{\mathrm{ei}}^{2}}{d}+\mathrm{CV}_{\mathrm{wb}}^{2}+\mathrm{CV}_{\mathrm{tp}}^{2}}$

$\mathrm{CV}_{\mathrm{e}}$

23

2

$\mathrm{CV}_{\mathrm{wb}}$
8.5

$\mathrm{CV}_{\mathrm{tp}}$

15

PAL

Under-reporters $\quad 0.96$

$2 \cdot 49$
1.55

40 out of $202(19 \%)$

45 out of $202(22 \%)$

48 out of $208(23 \%)$

$\mathrm{CM}$ risk 1, cardiometabolic risk score described by Alberti et al. ${ }^{(59)} ; \mathrm{N}_{-}$, normalised value with Bloom technique (similar to $z$ scores but all values go from -1 to 1 ). $\mathrm{N}$ inverted HDL, values of normalised HDL multiplied by -1 ; CM risk 2, cardiometabolic risk score created by Martinez Vizcaino et al. ${ }^{(58)}$; MAP, mean arterial blood pressure; Lazzer 1, Lazzer equation taking into account body composition; FFM, fat-free mass; FM, fat mass; Lazzer 2, Lazzer equation taking into account weight; BW, body weight; EI rep, reported energy

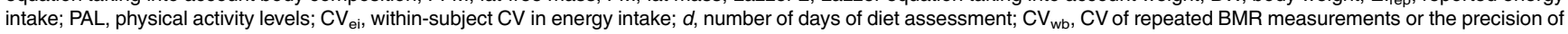
estimated compared with measured $\mathrm{BMR} ; \mathrm{CV}_{\mathrm{tp}}$, total variation in PAL.

and standard deviations are presented for the whole sample and stratified by sex.

Power calculation and sample size estimations were computed based on the primary outcome in each of the studies which are reported in the corresponding methodological articles published elsewhere ${ }^{(26,27)}$. The present study is based on a secondary analysis using baseline data from both studies, and therefore a specific power calculation was not developed for the present study.

Linear regression analyses were performed to explore the influence of $\mathrm{ED}$ on body composition and $\mathrm{CM}$ risk after adjustment for total EI, age, study centre, sex and mothers' educational level. Further models were explored taking MVPA into account. MVPA by ED interactions were tested to determine whether MVPA influenced the association between ED and the outcome variables. All the previously mentioned regressions were also performed, stratifying participants according to compliance or no compliance with physical activity recommendations (sixty daily minutes or more of $\mathrm{MVPA}^{(43)}$ ).

A sensitivity analysis was conducted excluding underreporters who were detected by the Goldberg method ${ }^{(31)}$ using three different equations ${ }^{(33,34)}$ as shown in Table 1. Dietary differences between under- and plausible-reporters were evaluated with independent $t$ tests (using under-reporters estimated with the Schofield equation ${ }^{(34)}$ as it was the most used equation in the literature).

Cluster analysis was performed with $\mathrm{ED}_{\mathrm{S}}$ and minutes of MVPA. To be consistent with clustering methods reported in previous studies ${ }^{(44,45)}$, two types of cluster analyses were used: hierarchical clustering (Ward's method) and $k$-means clustering. To reduce the sensitivity of the Ward's method to outliers, individual outliers and multivariate outliers (those with high Mahalanobis'distance values) for any variable were investigated. Hierarchical cluster analysis was initially used, as the numbers of clusters in the data were unknown beforehand.
Number of clusters was determined by examining dendrograms, suggesting a solution for three cluster groups. $k$-Means cluster analysis was therefore performed with three possible solutions. This approach minimises the within-cluster variance and maximises the between-cluster distance, so that the resulting clusters are as homogeneous as possible. $k$-Means cluster analysis is considered superior to hierarchical methods because it is less sensitive to outliers and has been found to result in greater within-cluster homogeneity and betweencluster heterogeneity ${ }^{(46)}$

One-way ANOVA with ED and MVPA variables were performed to classify and name the three cluster groups that emerged, that were described as healthy (low ED and high MVPA), inactive-low-ED (low ED and low MVPA) and averageMVPA-high-ED (high ED and medium MVPA). Finally, age, sex, study centre, total EI and mothers' education-adjusted ANCOVA were performed to evaluate adiposity and cardiometabolic risk differences among cluster groups.

\section{Results}

\section{Descriptive characteristics}

Participant characteristics, dietary variables, body composition values, cardiometabolic risk markers and physical activity levels are detailed in Table 2 . Briefly, the sample was 10.4 years old, with a mean BMI of $26 \cdot 1 \mathrm{~kg} / \mathrm{m}^{2}$, waist circumference of $84 \mathrm{~cm}$ and $41 \mathrm{BF} \%$. Regarding dietary habits, participants reported an EI of 7284 daily kJ (1741 kcal) with an average ED $_{S}$ of $7.6 \mathrm{~kJ} / \mathrm{g}$ $(1.81 \mathrm{kcal} / \mathrm{g})$ and $\mathrm{ED}_{\mathrm{SL}}$ of $9.5 \mathrm{~kJ} / \mathrm{g}(2.28 \mathrm{kcal} / \mathrm{g})$. BMR varied slightly depending on the used formula ranging from $5996 \mathrm{~kJ}$ (1433 kcal) with the Lazzer ${ }_{\text {DxA }}$ to $6204 \mathrm{~kJ}$ (1483 kcal) with the Schofield equation.

Data for the comparison between under-reporters and plausible-reporters are presented in online Supplementary 
Table 2. Characteristics of the whole sample and stratified according to sex and study

(Mean values and standard deviations)

\begin{tabular}{|c|c|c|c|c|c|c|c|c|c|c|}
\hline & \multicolumn{2}{|c|}{ Whole sample ( $n$ 208) } & \multicolumn{2}{|c|}{ Boys $(n 111)$} & \multicolumn{2}{|c|}{ Girls $(n 97)$} & \multicolumn{2}{|c|}{ EFIGRO $(n$ 107) } & \multicolumn{2}{|c|}{ Activebrains $(n$ 101) } \\
\hline & Mean & SD & Mean & SD & Mean & SD & Mean & SD & Mean & SD \\
\hline \multicolumn{11}{|l|}{ Descriptive characteristics } \\
\hline Age (years) & $10 \cdot 4$ & 1.2 & 10.5 & 1.2 & $10 \cdot 2$ & 1.1 & $10 \cdot 6$ & 1.1 & $10 \cdot 0$ & 1.1 \\
\hline Weight (kg) & 55.5 & $10 \cdot 7$ & $56 \cdot 4$ & $10 \cdot 3$ & 54.5 & $11 \cdot 0$ & 54.8 & $10 \cdot 6$ & $56 \cdot 1$ & $10 \cdot 7$ \\
\hline Height $(\mathrm{cm})$ & $145 \cdot 2$ & $8 \cdot 1$ & $145 \cdot 9$ & $8 \cdot 1$ & 144.5 & $8 \cdot 1$ & $146 \cdot 3$ & 8.0 & $144 \cdot 2$ & 8.2 \\
\hline Tanner stage $(\mathrm{I} / \mathrm{II} / \mathrm{III} / \mathrm{IV} / \mathrm{V})^{\star}$ & \multicolumn{2}{|c|}{$78 / 74 / 35 / 13 / 2$} & \multicolumn{2}{|c|}{$50 / 43 / 10 / 3 / 0$} & \multicolumn{2}{|c|}{$28 / 31 / 25 / 10 / 2$} & \multicolumn{2}{|c|}{$41 / 28 / 20 / 10 / 2$} & \multicolumn{2}{|c|}{$37 / 46 / 15 / 3 / 0$} \\
\hline Mothers education (low/medium/high) & \multicolumn{2}{|c|}{ 29/101/78 } & \multicolumn{2}{|c|}{$14 / 56 / 40$} & \multicolumn{2}{|c|}{$14 / 45 / 38$} & \multicolumn{2}{|c|}{$3 / 52 / 52$} & \multicolumn{2}{|c|}{$26 / 49 / 26$} \\
\hline \multicolumn{11}{|l|}{ Dietary variables } \\
\hline Energy intake (kJ/d) & 7284 & 1657 & 7556 & 1673 & 7021 & 1544 & 7615 & 1711 & 6983 & 1485 \\
\hline Total $\mathrm{CHO}(\mathrm{g} / \mathrm{d})$ & 182 & 49 & 188 & 46 & 176 & 51 & 192 & 52 & 172 & 43 \\
\hline Total fat $(\mathrm{g} / \mathrm{d})$ & 77 & 24 & 80 & 25 & 75 & 23 & 82 & 26 & 74 & 21 \\
\hline Total protein $(\mathrm{g} / \mathrm{d})$ & 76 & 19 & 81 & 20 & 71 & 17 & 77 & 20 & 76 & 18 \\
\hline Solids ED (kJ/g) & 7.53 & 1.76 & 7.53 & 1.72 & 7.57 & 1.80 & 8.08 & 1.84 & 7.03 & 1.46 \\
\hline Solids and liquids ED (kJ/g) & 4.77 & 0.88 & 4.81 & 0.88 & 4.77 & 0.88 & 5.02 & 0.92 & 4.56 & 0.79 \\
\hline \multicolumn{11}{|l|}{ Body composition } \\
\hline Body fat percentage & 41.7 & 5.5 & 41.0 & $5 \cdot 2$ & $42 \cdot 6$ & 5.7 & 39.5 & 4.6 & $44 \cdot 1$ & $5 \cdot 3$ \\
\hline Fat mass index $\left(\mathrm{kg} / \mathrm{m}^{2}\right)$ & 10.9 & $2 \cdot 7$ & $10 \cdot 8$ & $2 \cdot 7$ & $11 \cdot 1$ & $2 \cdot 7$ & $10 \cdot 1$ & $2 \cdot 4$ & 11.8 & $2 \cdot 8$ \\
\hline Abdominal FMI $\left(\mathrm{kg} / \mathrm{m}^{2}\right) \dagger$ & 0.81 & 0.30 & 0.79 & 0.29 & 0.81 & 0.31 & 0.72 & 0.27 & 0.91 & 0.31 \\
\hline Waist circumference $(\mathrm{cm})$ & 84.43 & $10 \cdot 19$ & 86.65 & 9.55 & 81.89 & $10 \cdot 34$ & $79 \cdot 0$ & $7 \cdot 6$ & $90 \cdot 2$ & $9 \cdot 4$ \\
\hline BMI $\left(\mathrm{kg} / \mathrm{m}^{2}\right)$ & $26 \cdot 1$ & 3.5 & $26 \cdot 3$ & 3.4 & $25 \cdot 9$ & 3.5 & $25 \cdot 5$ & 3.3 & $26 \cdot 8$ & 3.5 \\
\hline \multicolumn{11}{|l|}{ Cardiometabolic markers } \\
\hline Systolic BP (mmHg)‡ & $106 \cdot 4$ & 14.8 & $107 \cdot 7$ & 14.6 & $105 \cdot 0$ & 14.9 & 96.7 & 9.9 & 117.5 & $11 \cdot 1$ \\
\hline Diastolic BP $(\mathrm{mmHg}) \ddagger$ & $66 \cdot 2$ & 9.9 & $66 \cdot 9$ & $10 \cdot 4$ & $65 \cdot 3$ & $9 \cdot 2$ & 61.9 & $8 \cdot 1$ & $70 \cdot 9$ & 9.4 \\
\hline MAP $(\mathrm{mmHg}) \ddagger$ & 79 & 10 & 80 & 10 & 78 & 10 & 73 & 7 & 86 & 8 \\
\hline TAG $(\mathrm{mmol} / \mathrm{l}) \S$ & 1.00 & 0.49 & 0.96 & 0.46 & 1.06 & 0.50 & 0.95 & 0.45 & 1.06 & 0.51 \\
\hline TAG:HDL ratioß & $2 \cdot 0$ & $2 \cdot 1$ & 1.8 & 1.2 & $2 \cdot 3$ & $2 \cdot 8$ & 1.8 & $1 \cdot 1$ & $2 \cdot 3$ & $2 \cdot 8$ \\
\hline Total cholesterol $(\mathrm{mmol} / \mathrm{l}) \S$ & 4.39 & 0.71 & 4.39 & 0.72 & 4.40 & $0 \cdot 70$ & 4.44 & 0.71 & $4 \cdot 35$ & 0.71 \\
\hline $\mathrm{HDL}(\mathrm{mmol} / \mathrm{l}) \S$ & 1.30 & 0.29 & $1 \cdot 34$ & 0.31 & 1.25 & 0.28 & $1 \cdot 30$ & 0.28 & 1.30 & 0.31 \\
\hline Glucose $(\mathrm{mmol} / \mathrm{l}) \S$ & 4.77 & 0.34 & 4.83 & 0.32 & 4.69 & 0.36 & 4.75 & 0.31 & 4.79 & 0.38 \\
\hline LDL (mmol/l)\| & 2.66 & 0.64 & 2.63 & 0.63 & $2 \cdot 68$ & 0.65 & $2 \cdot 70$ & 0.61 & 2.59 & 0.66 \\
\hline Insulin (pmol/l) $\ddagger$ & $90 \cdot 29$ & 48.61 & 83.34 & 41.67 & $97 \cdot 23$ & 55.56 & 83.34 & $34 \cdot 73$ & $90 \cdot 29$ & 55.56 \\
\hline \multicolumn{11}{|l|}{ Physical activity levels } \\
\hline MVPA (min/d) & $54 \cdot 3$ & $21 \cdot 2$ & 59.7 & $21 \cdot 8$ & $48 \cdot 2$ & $18 \cdot 7$ & $56 \cdot 8$ & 21.9 & $51 \cdot 7$ & $20 \cdot 2$ \\
\hline \multicolumn{11}{|l|}{ Resting metabolic rate $(\mathrm{kcal}) \boldsymbol{\Phi}$} \\
\hline BMR Lazzer1* & 1462 & 188 & 1573 & 148 & 1340 & 147 & 1441 & 189 & 1483 & 190 \\
\hline BMR Lazzer $2^{*}$ & 1433 & 171 & 1535 & 130 & 1319 & 136 & 1422 & 171 & 1423 & 171 \\
\hline BMR Schofield & 1483 & 234 & 1641 & 183 & 1303 & 152 & 1457 & 232 & 1510 & 244 \\
\hline
\end{tabular}

$\mathrm{CHO}$, carbohydrates; ED, energy density; FMI, fat mass index; BP, blood pressure; MAP, mean arterial pressure; MVPA, moderate and vigorous physical activity; Lazzer 1, Lazzer equation calculated with Tanner stage and height; Lazzer 2, Lazzer equation calculated with fat percentage from dual-energy X-ray absorptiometry.

* $n 202$ (96 girls and 106 boys).

$\dagger n 190$ (85 girls and 105 boys)

$\ddagger n 201$ (94 girls and 107 boys)

$\S n 204$ (94 girls and 110 boys).

II $n 195$ (88 girls and 197 boys).

I To convert kcal to kJ, multiply by $4 \cdot 184$.

Table S1. Under-reporters presented lower values of total EI (5640 v. $7849 \mathrm{~kJ}(1348 v .1876 \mathrm{kcal}) ; P<0 \cdot 001), \mathrm{ED}_{\mathrm{S}}(6 \cdot 69 v .7 .82$ $\mathrm{kJ} / \mathrm{g}(1.6 v .1 .87 \mathrm{kcal} / \mathrm{g}) ; P<0.001)$ and $\mathrm{ED}_{\mathrm{SL}}(4.31 v .4 .90 \mathrm{~kJ} / \mathrm{g}$ (1.03 v. $1.17 \mathrm{kcal} / \mathrm{g}) ; P<0.001)$ when compared to plausible reporters. For all the body composition variables, underreporters presented higher values than plausible reporters (online Supplementary Table S1).

\section{Associations between dietary energetic density, body composition and cardiovascular risk}

Neither $\mathrm{ED}_{\mathrm{S}}$ nor $\mathrm{ED}_{\mathrm{SL}}$ demonstrated significant associations with any of the body composition or cardiovascular risk variables when the whole sample was analysed (Tables 3 and 4).

When under-reporters were excluded (Table 3), EDs was significantly associated with BF\% (Lazzer ${ }_{\text {DxA }} P=0.013$, Lazzer $_{\text {weight }} P=0.008$, Schofield $P=0.005$ ), FMI (Lazzer ${ }_{\text {DXA }}$
$P=0.025$, Lazzer $_{\text {weight }} P=0 \cdot 014$, Schofield $\left.P=0 \cdot 008\right)$ and abdominal FMI (Lazzer ${ }_{\text {DXA }} P=0 \cdot 040$, Lazzer $_{\text {weight }} P=0.033$, Schofield $P=0 \cdot 018$ ). These results remained significant after adjusting for MVPA (Table 3, model 2). $\mathrm{ED}_{\mathrm{s}}$ was also significantly associated with BMI when the Schofield $(P=0 \cdot 019)$ and Lazzer $_{\text {weight }}(P=0.036)$ equations were used to detect under-reporters and remained significant after adjusting for MVPA (Table 3, model 2). A tendency was also found for the Lazzer $_{\text {DXA }}$ equation when predicting BMI $(P=0.053$ and $P=0.067$ without and with adjustment of MVPA, respectively).

Regarding the association between $\mathrm{ED}_{\mathrm{SL}}$ and body composition when under-reporters were excluded (Table 4), ED $\mathrm{SL}_{\mathrm{S}}$ was significantly associated with $\mathrm{BF} \%$ (Lazzer $_{\mathrm{DXA}} P=0.031$, Lazzer $_{\text {weight }} P=0.017$, Schofield $\left.P=0.008\right)$ even after adjusting for MVPA (Table 4, model 2). $\mathrm{ED}_{\mathrm{SL}}$ was also associated with FMI when under-reporters were detected with the Schofield $(P=0.026)$ and the Lazzer $_{\text {weight }}(P=0.040)$ 
Table 3. Linear regressions testing the influence of energetic density (only solids) on body composition variables and cardiometabolic (CM) risk scores

\begin{tabular}{|c|c|c|c|c|c|c|c|c|c|c|c|}
\hline Sample & BMR equation & Independent var. & & & $\mathrm{BMI}$ & $\begin{array}{l}\text { Waist } \\
\text { circumference }\end{array}$ & $\mathrm{BF} \%$ & FMI & ABD_FMI & $\mathrm{CM}$ risk 1 & $\mathrm{CM}$ risk 2 \\
\hline \multirow[t]{5}{*}{ Whole sample } & Not used & Energetic density & & $n$ & 208 & 208 & 208 & 208 & 190 & 196 & 191 \\
\hline & & & M1 & $B$ standard & 0.064 & 0.057 & $0 \cdot 107$ & 0.081 & 0.095 & -0.041 & 0.014 \\
\hline & & & & $P$ & 0.389 & 0.364 & 0.163 & 0.298 & 0.251 & 0.604 & 0.861 \\
\hline & & & M2 & $B$ standard & 0.044 & 0.043 & 0.087 & 0.060 & 0.081 & -0.063 & -0.004 \\
\hline & & & & $P$ & 0.536 & 0.482 & 0.238 & 0.418 & 0.306 & 0.408 & 0.953 \\
\hline \multirow{15}{*}{$\begin{array}{l}\text { Under-reporters } \\
\text { excluded }\end{array}$} & Lazzer $_{\mathrm{DXA}}$ & Energetic density & & $n$ & $162 \dagger$ & 162 & $162^{*}$ & $162^{*}$ & $149^{*}$ & 154 & 151 \\
\hline & & & M1 & $B$ standard & $0.162 \dagger$ & 0.092 & $0.208^{*}$ & $0.193^{*}$ & $0.188^{*}$ & -0.038 & 0.037 \\
\hline & & & & $P$ & $0.053 \dagger$ & 0.196 & $0.013^{\star}$ & $0.025^{\star}$ & $0.040^{*}$ & 0.672 & 0.666 \\
\hline & & & M2 & $B$ standard & $0.148 \dagger$ & 0.084 & $0.194^{*}$ & $0.179^{*}$ & $0.183^{*}$ & -0.047 & 0.029 \\
\hline & & & & $P$ & $0.067 \dagger$ & 0.232 & $0.017^{\star}$ & $0.033^{*}$ & $0.041^{\star}$ & 0.591 & 0.726 \\
\hline & Lazzer $_{\text {weight }}$ & Energetic density & M1 & $n$ & $157^{*}$ & 157 & $157^{\star}$ & $157^{\star}$ & $146^{\star}$ & 149 & 147 \\
\hline & & & & $B$ standard & $0.175^{\star}$ & 0.094 & $0.223^{*}$ & $0.211^{*}$ & $0.195^{\star}$ & -0.042 & 0.030 \\
\hline & & & & $P$ & $0.036^{*}$ & 0.183 & $0.008^{*}$ & $0.014^{*}$ & $0.033^{*}$ & 0.638 & 0.728 \\
\hline & & & M2 & $B$ standard & $0 \cdot 158^{*}$ & 0.083 & $0.204^{*}$ & $0.193^{*}$ & $0 \cdot 186^{*}$ & -0.055 & 0.019 \\
\hline & & & - & $P$ & $0.050^{*}$ & 0.233 & $0.012^{*}$ & $0.020^{*}$ & $0.036^{*}$ & 0.528 & 0.822 \\
\hline & Schofield & Energetic density & M1 & $n$ & $157^{\star}$ & 157 & $157^{\star}$ & $157^{\star}$ & $145^{\star}$ & 149 & 149 \\
\hline & & & & $B$ standard & $0 \cdot 198^{*}$ & 0.121 & $0.237^{*}$ & $0.228^{*}$ & $0 \cdot 216^{*}$ & -0.014 & 0.062 \\
\hline & & & & $P$ & $0.019^{\star}$ & 0.098 & $0.005^{*}$ & $0.008^{*}$ & $0.018^{*}$ & 0.874 & 0.474 \\
\hline & & & M2 & $B$ standard & $0 \cdot 181^{*}$ & 0.111 & $0.218^{*}$ & $0.209^{*}$ & $0.206^{*}$ & -0.029 & 0.051 \\
\hline & & & & $P$ & $0.028^{\star}$ & 0.125 & $0.007^{\star}$ & $0.012^{*}$ & $0.021^{\star}$ & 0.743 & 0.551 \\
\hline
\end{tabular}

BF\%, body fat percentage; FMI, fat mass index; ABD_FMI, abdominal fat mass index; CM risk1, CM risk score described by Alberti et al..$^{(59)}$; CM risk 2, CM risk score created by Martinez Vizcaino et al. ${ }^{(58)}$; M1, model 1, adjusted by sex, age, mother's education and total energy intake; $\mathrm{M} 2$, model $1+$ moderate and vigorous physical activity adjustment; DXA, dual-energy $\mathrm{X}$-ray absorptiometry.

$* \quad P \leq 0.050$.

$\dagger P<0.07$ 

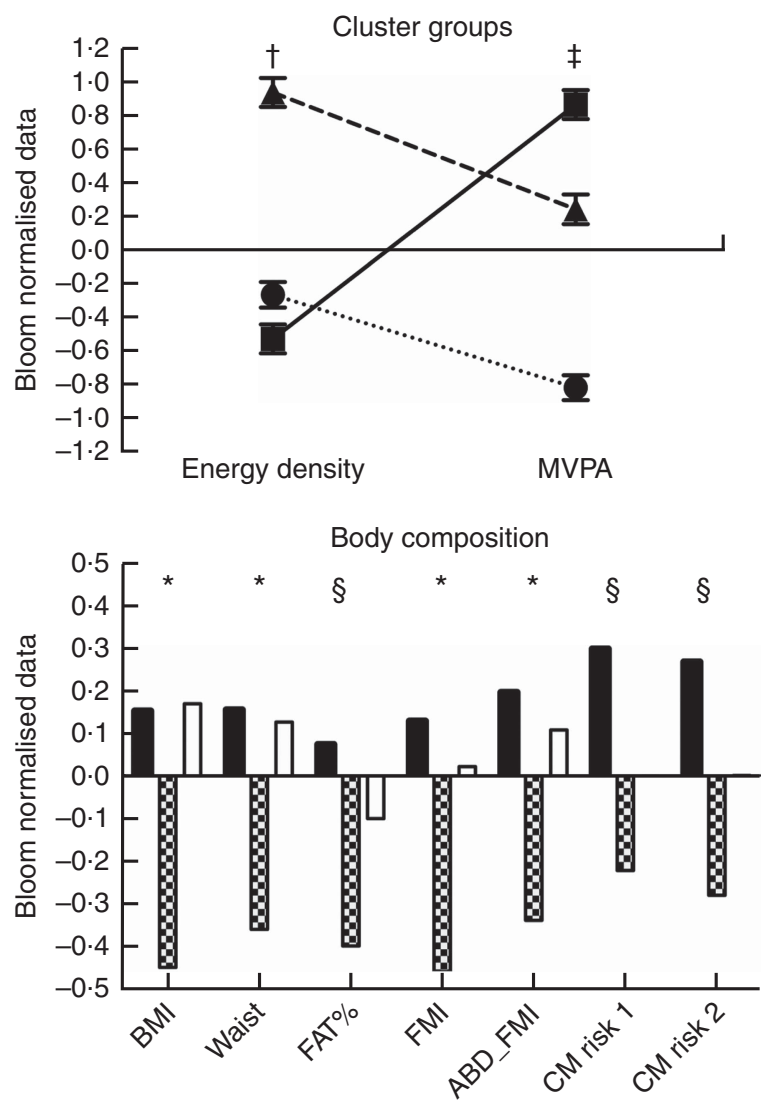

Fig. 1. Cluster analysis results. Body composition comparisons adjusted by mothers' education, study centre, sex, age and total energy intake. * Differences between the healthy group (low energy density (ED) and high moderate to vigorous physical activity (MVPA)) and the other two groups. † Differences between the average MVPA high ED and the other two groups. $\ddagger$ Differences among the three groups $(P<0.05)$. $\S$ Differences between the healthy group (low ED and high MVPA) and the inactive and low ED group. ABD_FMl, abdominal fat mass index; $\mathrm{CM}$ risk 2, cardiometabolic risk score created by Martinez Vizcaino et al. ${ }^{(58)}$; CM risk 1 , cardiometabolic risk score described by Alberti et al. ${ }^{(59)}$; FAT\%, body fat percentage; FMI, fat mass index.

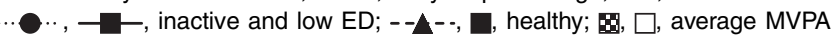
and high ED.

equations, but only results for the Schofield equation remained significant after adjusting for MVPA $(P=0.042$ for the Schofield equation and $P=0.055$ for the Lazzer $_{\text {weight }}$ equation, Table 3, model 2).

No significant interactions were found between MVPA and ED in the performed linear regression models $(P>0 \cdot 10)$ for the whole sample. Nonetheless when under-reporters were excluded, the interaction terms for BF\% (Schofield $P=0.049$ ) became significant. When the whole sample was stratified according to compliance with physical activity recommendations, no association was found between ED and any of the outcome variables for compliers ( $n$ 73) and non-compliers ( $n$ 135). When under-reporters were excluded, different results were found for compliers ( $n$ 63, 62 and 62 for the Lazzer ${ }_{\text {DXA }}$, Lazzer $_{\text {weight }}$ and Schofield equations, respectively) and non-compliers ( $n$ 99, 95

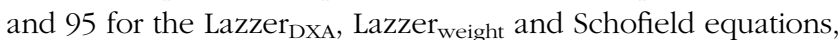
respectively). For the complier group, no significant association was found between ED and any of the outcome variables, while for the non-complier group positive association was found between ED, BF\% (Lazzer ${ }_{\text {DXA }} P=0 \cdot 016$, Lazzer $_{\text {weight }} P=0 \cdot 011$ and Schofield $P=0.004$ ) and FMI (Lazzer ${ }_{\text {DxA }} P=0.080$, Lazzer $_{\text {weight }} P=0.060$ and Schofield $P=0.024$ ). Additional adjustment by MVPA did not modify results of the stratified aforementioned regressions.

\section{Combined effect of energy density and moderate to vigorous physical activity: cluster analysis}

Finally, when analysing the cluster analysis results (Fig. 1), we observed that the healthy group (low ED and high MVPA levels) presented lower values of BMI (both $P<0.001$ ), waist circumference (both $P<0.001)$, FMI $(P<0.001 v$. inactive-low-ED and $P=0.006 \quad v$. average-MVPA-high-ED) and abdominal FMI $(P=0.003 v$. inactive-low-ED and $P=0.015 v$. averageMVPA-high-ED) when compared to the other two groups. The healthy group also presented lower values than the inactivelow-ED group for both estimated cardiovascular risk indexes (cardiorisk-1 $P=0.005$; cardiorisk-2 $\quad P=0.002$ ) and $\quad \mathrm{BF} \%$ $(P=0.003)$. No differences were found between inactive-lowED and average-MVPA-high-ED for any of the measured variables (all $P>0 \cdot 05$; Fig. 1).

\section{Discussion}

The main findings of the present study are (1) dietary ED is associated with higher total and abdominal adiposity in plausible reporters of dietary $\mathrm{EI}$, with $\mathrm{ED}_{\mathrm{S}}$ showing stronger associations than $\mathrm{ED}_{\mathrm{SL}}$, (2) the aforementioned association persists even after adjusting for MVPA. Nonetheless, when stratifying plausible reporters according to complying or not complying with physical activity recommendations, the inverse association between ED and adiposity disappears for the complying group and is maintained for the non-complying group. Those children with overweight/obesity with a combination of high MVPA levels and low dietary ED present a healthier body composition and cardiometabolic profile and (3) the effect of excluding under-reporters from the analysis is critical in the study of children with overweight and obesity. Although previous literature is inconsistent, the influence of dietary ED on body composition when under-reporters were excluded in our sample was clear, and we believe that inconsistencies found among previous studies ${ }^{(8)}$ could partly be explained by some of the findings discussed below.

On the one hand, it becomes clear that $\mathrm{ED}_{\mathrm{S}}$ and $\mathrm{ED}_{\mathrm{SL}}$ do not show the same association with adiposity in overweight and obese children, as only $\mathrm{ED}_{\mathrm{S}}$ significantly predicted $\mathrm{BMI}$ and abdominal FMI. This is in line with previous studies performed with participants without obesity, which also found that ED calculated with only solids demonstrated better association with body composition in children ${ }^{(17,47)}$ and adults ${ }^{(48)}$ compared to ED calculated with solids and liquids. We thought it was important to evaluate whether the differences in ED calculation also emerged when evaluating children with overweight and obesity, as their intake of sugar-sweetened drinks might be higher, which, in turn, could modify the association between $\mathrm{ED}_{\mathrm{SL}}$ and body composition. Differences between the 
Table 4. Linear regressions testing the influence of energetic density (solids and liquids) on body composition variables and cardiometabolic (CM) risk scoresł

\begin{tabular}{|c|c|c|c|c|c|c|c|c|c|c|c|}
\hline Sample & BMR equation & Independent var. & & & BMI & $\begin{array}{l}\text { Waist } \\
\quad \text { circumference }\end{array}$ & $\mathrm{BF} \%$ & FMI & ABD_FMI & $\mathrm{CM}$ risk 1 & $\mathrm{CM}$ risk 2 \\
\hline \multirow[t]{5}{*}{ Whole sample } & \multirow[t]{5}{*}{ Not used } & \multirow[t]{5}{*}{ Energetic density } & \multirow[t]{3}{*}{ M1 } & $n$ & 208 & 208 & 208 & 208 & 190 & 196 & 191 \\
\hline & & & & $B$ standard & 0.004 & 0.002 & 0.067 & 0.026 & 0.013 & -0.097 & -0.033 \\
\hline & & & & $P$ & 0.957 & 0.979 & 0.387 & 0.736 & 0.871 & 0.213 & 0.673 \\
\hline & & & \multirow[t]{2}{*}{ M2 } & $B$ standard & -0.017 & -0.013 & 0.045 & 0.004 & -0.003 & $-0 \cdot 122$ & -0.053 \\
\hline & & & & $P$ & 0.808 & 0.827 & 0.541 & 0.952 & 0.974 & 0.107 & 0.471 \\
\hline \multirow{15}{*}{$\begin{array}{l}\text { Under-reporters } \\
\text { excluded }\end{array}$} & \multirow{5}{*}{ Lazzer $_{\text {DXA }}$} & \multirow{5}{*}{ Energetic density } & \multirow[t]{3}{*}{ M1 } & $n$ & 162 & 162 & $162^{*}$ & 162 & 149 & 154 & 151 \\
\hline & & & & $B$ standard & 0.120 & 0.040 & $0.182^{*}$ & 0.152 & 0.122 & -0.131 & -0.006 \\
\hline & & & & $P$ & 0.149 & 0.569 & $0.031^{*}$ & 0.077 & 0.179 & 0.274 & 0.940 \\
\hline & & & \multirow[t]{2}{*}{ M2 } & $B$ standard & 0.105 & 0.031 & $0 \cdot 165^{*}$ & 0.136 & 0.298 & $-0 \cdot 108$ & -0.017 \\
\hline & & & & $P$ & 0.195 & 0.656 & $0.042^{*}$ & 0.103 & 0.199 & 0.214 & 0.841 \\
\hline & \multirow[t]{5}{*}{ Lazzer $_{\text {weight }}$} & \multirow[t]{5}{*}{ Energetic density } & \multirow[t]{3}{*}{ M1 } & $n$ & 157 & 157 & $157^{\star}$ & $157^{\star}$ & 146 & 149 & 147 \\
\hline & & & & $B$ standard & 138 & 0.050 & $0.202^{*}$ & $0 \cdot 178^{*}$ & 0.133 & -0.092 & -0.006 \\
\hline & & & & $P$ & 0.098 & 0.483 & $0.017^{\star}$ & $0.040^{*}$ & 0.145 & 0.299 & 0.943 \\
\hline & & & \multirow[t]{2}{*}{ M2 } & $B$ standard & 0.121 & 0.038 & $0.184^{*}$ & $0.159 \dagger$ & 0.122 & -0.106 & -0.018 \\
\hline & & & & $P$ & 0.133 & 0.580 & $0.023^{*}$ & $0.055 \dagger$ & 0.167 & 0.222 & 0.825 \\
\hline & \multirow[t]{5}{*}{ Schofield } & \multirow[t]{5}{*}{ Energetic density } & \multirow[t]{3}{*}{ M1 } & $n$ & 157 & 157 & $157^{\star}$ & $157^{\star}$ & 145 & 149 & 149 \\
\hline & & & & $B$ standard & 0.153 & 0.072 & $0.222^{*}$ & $0 \cdot 191^{*}$ & 0.150 & -0.069 & 0.019 \\
\hline & & & & $P$ & 0.071 & 0.329 & $0.008^{*}$ & $0.026^{*}$ & 0.099 & 0.434 & 0.823 \\
\hline & & & \multirow[t]{2}{*}{ M2 } & $B$ standard & 0.133 & 0.060 & $0.201^{*}$ & $0 \cdot 170^{*}$ & 0.136 & -0.087 & 0.005 \\
\hline & & & & $P$ & 0.108 & 0.409 & $0.013^{\star}$ & $0.042^{*}$ & 0.126 & 0.316 & 0.956 \\
\hline
\end{tabular}

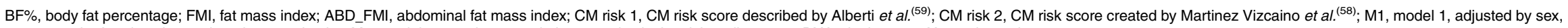
age, mother's education and total energy intake; M2, model $1+$ moderate and vigorous physical activity adjustment; DXA, dual-energy X-ray absorptiometry.

${ }^{*} P \leq 0.050$.
$+P<0.07$.

¥ All models adjusted by sex, age, mother's education and total energy intake. 
association of $\mathrm{ED}_{\mathrm{S}}$ and $\mathrm{ED}_{\mathrm{SL}}$ with body composition found in the present and in previous studies could be due to the different effects that drinks and solid foods have on satiety ${ }^{(49)}$ and to the differences in ED between solids and liquids, as beverages tend to have a high water content and low ED. Consequently, the inclusion of beverages in ED calculations will lower the ED of the entire meal and bias the association between ED and body composition towards the null as seen in our and previous studies $^{(17,48)}$

On the other hand, we found that the association between ED in plausible reporters and adiposity was only significant for some variables; likewise, $\mathrm{ED}_{\mathrm{S}}$ was associated with $\mathrm{BF} \%$, FMI and abdominal FMI, all measured with DXA, but did not show an association with less accurate proxies of body fat such as waist circumference or BMI (when excluding under-reporters with the Lazzer ${ }_{\text {DXA }}$ equation). Regarding the association of ED and these proxies, literature is inconsistent, as some studies show positive associations ${ }^{(21,50)}$, while others do $\operatorname{not}^{(14,51)}$. Nonetheless, these are just proxies, with the measurement of body fat being more important. To the best of our knowledge, the only previous study that evaluated ED and body composition of children with obesity with DXA was the Viva la familia study $^{(12)}$. This study used total body mass gain (during 1 year follow-up) as an outcome variable, finding that ED did not significantly predict weight gain. DXA variables (fat mass and fat-free mass) were not used as outcome variables in the aforementioned study and, therefore, comparisons with our findings in children with overweight/obesity are not possible. Regarding children without obesity, three previous studies evaluated the influence of ED on body fat measured with DXA, with two of them reporting positive association between both variables $^{(11,52)}$, and one developed by Kral et al. ${ }^{(18)}$ not finding significant associations. Kral et al. suggested that sample size ( $n$ 49) might have been too small to detect significant association between the two variables. Moreover, although 3-d weighed food records were used in the previous study, there was no control for under-reporters that have shown to affect the results in our and previous studies ${ }^{(15,23,53)}$.

The effect of excluding the under-reporters from the analysis was large in the present sample. They presented higher adiposity values, $27 \%$ lower EI, $18 \%$ lower levels of MVPA and more importantly a significantly lower ED when compared to plausible reporters, as published previously ${ }^{(53)}$. This lower ED found in under-reporters could bias the association between ED and body composition variables towards the null if not accounted for in the analysis, explaining why no association was found between ED and body composition variables when the whole sample was analysed. The findings of MVPA not modifying the association between ED and body composition (only FMI for one equation passed from $P 0.040$ to $P$ 0.055) are of great importance, as it demonstrates that ED is crucial for children with overweight and obesity even when taking into account their levels of physical activity. Nonetheless, this does not mean that MVPA is not determinant of adiposity in this population, as when the sample was stratified according to compliance with physical activity recommendations, no significant association was found between ED and body composition or cardiovascular risk variables in the complier group.
Moreover, from the cluster analysis, it became clear that those who performed the highest levels of MVPA and showed low levels of ED presented the healthier profile for all the measured variables.

In agreement with a previous study ${ }^{(15)}$, ED was not directly associated with cardiometabolic risk in children. This lack of association could be partly explained by the characteristics of the measured sample, a homogeneous group of overweight and obese children who showed high cardiometabolic marker values when compared to previous studies ${ }^{(36)}$ using the same methodology in normal-weight children (systolic blood pressure $106 v$. 99, diastolic blood pressure $66 v$. 61, TAG $89 v$. 61, HDL $50 v$. 62, LDL $103 v .95$ in our study $v$. Nyström et al. ${ }^{(36)}$ study, respectively). It is possible that if a more heterogeneous sample of both children with normal weight and overweight had been included, significant associations between ED and cardiometabolic risk would have been found, as seen in previous studies that included both normal weight and overweight children $^{(54)}$. Although the associations between ED and cardiometabolic risk were non-significant, in the cluster analysis, the healthy group presented significantly lower cardiometabolic risk than the inactive-low-ED, and no differences with the average-MVPA-high-ED. These findings suggest that MVPA levels in children with obesity might be determinant of cardiometabolic risk, which is in line with previous studies that underlined the importance of physical activity on cardiometabolic risk factors in children ${ }^{(5)}$

Although the current study presents several strengths such as the measurement of objective physical activity, body fat with DXA and collection of cardiometabolic risk markers, it also presents several limitations. First, $24 \mathrm{~h}$ recalls were only collected for weekdays, and the photographic manual used to help participants select portion sizes is still in the process of being validated. It is therefore important to notice that results could be biased by the previously mentioned tool and by the use of only weekdays, as previous research has demonstrated important differences between week and weekend days ${ }^{(56)}$. Second, the cross-sectional nature of the study does not allow for a causeeffect conclusion, and although we found associations, these do not imply that a higher ED intake will necessarily mean an increase in the measured body composition variables. Moreover, body composition assessments were performed during the morning in a non-fasted state, which could affect the quantification of lean and fat mass ${ }^{(57)}$. Third, non-energycontaining beverages, such as water, tea or diet soda, were not included in the ED calculations and therefore the findings of the present study could have been different if the whole diet of each participant had been taken into account. Finally, although the present study included a homogeneous sample of 8- to 12year-old overweight and obese children, the sample size was smaller compared to the previous similar studies ${ }^{(14,21)}$ that included heterogeneous samples. This sample size could entail a low power and a type II error, and therefore it is possible that if a larger sample size had been analysed further associations would have been found between ED and cardiometabolic markers.

Summarising, findings from the present study suggest that dietary ED is associated with total and abdominal adiposity in 
children with overweight and obesity when under-reporters are excluded from the analysis. The large inconsistencies among previous studies could be due to the use of different ED calculations, the use of different methodologies to estimate body fat mass and under-reporting concerns (as for the present study, most results were modified when excluding underreporters). Those overweight and obese participants who performed high levels of MVPA and presented a low ED intake demonstrated the healthier profile for both body composition and cardiometabolic risk variables. In conclusion, findings from the present study suggest that children with overweight or obesity with a low ED intake and high levels of MVPA present healthier body composition and cardiometabolic profiles when compared to children with overweight or obesity who perform lower amounts of MVPA and have a higher ED intake. Consequently, nutritional programs aiming to prevent or treat childhood obesity should try to avoid high $\mathrm{ED}_{\mathrm{S}}$ foods and promote physical activity. These findings might have important public health implications, yet they need to be confirmed by randomised controlled trials that target both diet density and MVPA.

\section{Acknowledgements}

We thank the participation of the children and parents in this study.

The research leading to these results has received funding from 'la Caixa' Foundation and Triptolemos Foundation, the Ministry of Health (FIS PI081297), the Research Network on Preventative Activities and Health Promotion (RD06/0018/ 0038), the Henning and Johan Throne-Holst Foundation (F. B. O.), the Spanish Ministry of Education, Culture and Sport (FPU14/03329 to M. M.), the Spanish Ministry of Economy and Competitiveness (DEP2013-47540 and DEP2016-78377-R; BES-2014-068829 to C. C.-S.), Fondo de Investigación Sanitaria del Instituto de Salud Carlos III (PI13/01335), Fondos Estructurales de la Unión Europea (FEDER), Una manera de hacer Europa, the Spanish Ministry of Science and Innovation (RYC-2011-09011 to F. B. O.), the University of Granada, Plan Propio de Investigación 2016, Excellence Actions: Units of Excellence, Unit of Excellence on Exercise and Health (UCEES), Programa de Captación de Talento - UGR Fellows (L. G.-M.), the SAMID III network, RETICS (PN I+D + I 2017-2021).

This study has been partially funded by the University of Granada, Plan Propio de Investigación 2016, Excellence actions: Units of Excellence; Unit of Excellence on Exercise and Health (UCEES), and by the Junta de Andalucía, Consejería de Conocimiento, Investigación y Universidades and European Regional Development Fund (ERDF), reference SOMM17/6107/UGR.

ISCIII-Sub-Directorate General for Research Assessment and Promotion, the European Regional Development Fund (RD16/ 0022), the EXERNET Research Network on Exercise and Health in Special Populations (DEP2005-00046/ACTI), and the University of the Basque Country (GIU14/21). J. M.-G. is supported by the Spanish Ministry of Education, Culture and Sport (FPU14/06837). J. H. M. is supported by the Spanish Ministry of Education, Culture and Sport (FPU15/02645).
L. A. received a grant from the Education Department of the Government of the Basque Country (PRE_2016_1_0057, PRE_2017_2_0224, PRE_2018_2_0057).

Collected the data: V. M.-H., J. M.-G., E. M.-R., M. O., J. H. M., L. A., M. M., W. D. M.-A., J. M., I. T., I. L. and F. B. O. Performed the statistical analyses and drafted the manuscript: A. G.-B. Reviewed the manuscript: L. A., M. M., J. M.-G., C. C.-S., J. H. M., V. M.-H., E. M.-R., W. D. M.-A., J. M., M. O., I. T., L. G.-M., G. V.-R., F. B. O. and I. L. Conceived and planned the experiments: F. B. O. and I. L. Coordinated the project: F. B. O., I. L., L. A. and G. V.-R. Approved the final version: A. G.-B., L. A., M. M., J. M.-G., C. C.-S., J. H. M., V. M.-H., E. M.-R., W. D. M.-A., J. M., M. O., I. T., L. G.-M., G. V.-R., F. B. O. and I. L.

On behalf of all authors, A. G.-B. states that there is no conflict of interest. All authors have nothing to disclose.

\section{References}

1. Geiß H, Parhofer K \& Schwandt P (2001) Parameters of childhood obesity and their relationship to cardiovascular risk factors in healthy prepubescent children. Int J Obes 25, 830-837.

2. Gibson LY, Allen KL, Davis E, et al. (2017) The psychosocial burden of childhood overweight and obesity: evidence for persisting difficulties in boys and girls. Eur J Pediatr 176, 925-933.

3. Taylor ED, Theim KR, Mirch MC, et al. (2006) Orthopedic complications of overweight in children and adolescents. Pediatrics 117, 2167-2174.

4. Singh AS, Mulder C, Twisk JWR, et al. (2008) Tracking of childhood overweight into adulthood: a systematic review of the literature. Obes Rev 9, 474-488.

5. Schwingshackl L, Hoffmann G, Kalle-Uhlmann T, et al. (2015) Fruit and vegetable consumption and changes in anthropometric variables in adult populations: a systematic review and metaanalysis of prospective cohort studies. PLOS ONE 10, e0140846.

6. Brauchla M, Juan W, Story J, et al. (2012) Sources of dietary fiber and the association of fiber intake with childhood obesity risk (in 2-18 year olds) and diabetes risk of adolescents 12-18 year olds: NHANES 2003-2006. J Nutr Metab 2012, 736258.

7. Luger M, Lafontan M, Bes-Rastrollo M, et al. (2017) Sugarsweetened beverages and weight gain in children and adults: a systematic review from 2013 to 2015 and a comparison with previous studies. Obes Facts 10, 674-693.

8. Rouhani MH, Haghighatdoost F, Surkan PJ, et al. (2016) Associations between dietary energy density and obesity: a systematic review and meta-analysis of observational studies. Nutrition 32, 1037-1047.

9. Alexy U, Sichert-Hellert W, Kersting M, et al. (2004) Pattern of long-term fat intake and BMI during childhood and adolescence - results of the DONALD study. Int J Obes 28, 1203-1209.

10. Alexy U, Libuda L, Mersmann S, et al. (2011) Convenience foods in children's diet and association with dietary quality and body weight status. Eur J Clin Nutr 65, 160-166.

11. Ambrosini GL, Emmett PM, Northstone K, et al. (2012) Identification of a dietary pattern prospectively associated with increased adiposity during childhood and adolescence. Int J Obes 36, 1299-1305.

12. Butte NF, Cai G, Cole SA, et al. (2007) Metabolic and behavioral predictors of weight gain in Hispanic children: the Viva la Familia Study. Am J Clin Nutr 85, 1478-1485.

13. Günther ALB, Stahl LJ, Buyken AE, et al. (2011) Association of dietary energy density in childhood with age and body fatness at the onset of the pubertal growth spurt. Br J Nutr 106, 345-349. 
14. Hebestreit A, Börnhorst C, Barba G, et al. (2014) Associations between energy intake, daily food intake and energy density of foods and BMI $z$-score in 2-9-year-old European children. Eur J Nutr 53, 673-681.

15. Donin AS, Nightingale CM, Owen CG, et al. (2014) Dietary energy intake is associated with type 2 diabetes risk markers in children. Diabetes Care 37, 116-123.

16. Skinner AC, Perrin EM, Moss LA, et al. (2015) Cardiometabolic risks and severity of obesity in children and young adults. N Engl J Med 373, 1307-1317.

17. McCaffrey TC, Rennie KL, Kerr MA, et al. (2008) Energy density of the diet and change in body fatness from childhood to adolescence; is there a relation? Am J Clin Nutr 87, 1230-1237.

18. Kral TVE, Berkowitz RI, Stunkard AJ, et al. (2007) Dietary energy density increases during early childhood irrespective of familial predisposition to obesity: results from a prospective cohort study. Int J Obes 31, 1061-1067.

19. Vernarelli JA, Mitchell DC, Hartman TJ, et al. (2011) Dietary energy density is associated with body weight status and vegetable intake in U.S. children. J Nutr 141, 2204-2210.

20. O'Sullivan TA, Bremner AP, Bremer HK, et al. (2015) Dairy product consumption, dietary nutrient and energy density and associations with obesity in Australian adolescents. I Hum Nutr Diet 28, 452-464.

21. Murakami K, Miyake Y, Sasaki S, et al. (2012) An energydense diet is cross-sectionally associated with an increased risk of overweight in male children, but not in female children, male adolescents, or female adolescents in Japan: the Ryukyus Child Health Study. Nutr Res 32, 486-494.

22. Shephard RJ. (2003) Limits to the measurement of habitual physical activity by questionnaires - Commentary. Br J Sports Med 37, 197-206.

23. Aburto TC, Cantoral A, Hernández-Barrera L, et al. (2015) Usual dietary energy density distribution is positively associated with excess body weight in Mexican children. $J$ Nutr 145, 1524-1530.

24. Gomes D, Luque V, Xhonneux A, et al. (2017) A simple method for identification of misreporting of energy intake from infancy to school age: results from a longitudinal study. Clin Nutr 37, 1053-1060.

25. Murakami K, Miyake Y, Sasaki S, et al. (2012) Characteristics of under- and over-reporters of energy intake among Japanese children and adolescents: the Ryukyus Child Health Study. Nutrition 28, 532-538.

26. Cadenas-Sánchez $\mathrm{C}$, Mora-González J, Migueles $\mathrm{JH}$, et al. (2016) An exercise-based randomized controlled trial on brain, cognition, physical health and mental health in overweight/obese children (ActiveBrains project): rationale, design and methods. Contemp Clin Trials 47, 315-324.

27. Medrano M, Maiz E, Maldonado-Martín S, et al. (2015) The effect of a multidisciplinary intervention program on hepatic adiposity in overweight-obese children: protocol of the EFIGRO study. Contemp Clin Trials 45, 346-355.

28. Cole TJ \& Lostein T (2012) Extended international (IOTF) body mass indez cut-offs for thiness, overweight and obesity. Pediatr Obes 7, 284-294.

29. Moliner-Urdiales D, Ruiz JR, Vicente-Rodriguez G, et al. (2011) Associations of muscular and cardiorespiratory fitness with total and central body fat in adolescents: the HELENA study. Br J Sports Med 45, 101-108.

30. EFSA (2014) Guidance on the EU menu methodology. EFSA J 12, 3944.

31. Goldberg GR, Black AE, Jebb SA, et al. (1991) Critical evaluation of energy intake data using fundamental principles of energy physiology: 1 . Derivation of cut-off limits to identify under-recording. Eur J Clin Nutr 45, 569-581.
32. Black AE (2000) Critical evaluation of energy intake using the Goldberg cut-off for energy intake: basal metabolic rate. A practical guide to its calculation, use and limitations. Int J Obes Relat Metab Disord 24, 1119-1130.

33. Lazzer S, Patrizi A, De Col A, et al. (2014) Prediction of basal metabolic rate in obese children and adolescents considering pubertal stages and anthropometric characteristics or body composition. Eur J Clin Nutr 68, 695-699.

34. Schofield WN (1985) Predicting basal metabolic rate, new standards and review of previous work. Hum Nutr Clin Nutr 39, Suppl. 1, 5-41.

35. Tooze JA, Krebs-Smith SM, Troiano RP, et al. (2012) The accuracy of the Goldberg method for classifying misreporters of energy intake on a food frequency questionnaire and 24-h recalls: comparison with doubly labeled water. Eur J Clin Nutr 66, 569-576.

36. Nyström CD, Henriksson P, Martínez-Vizcaíno V, et al. (2017) Does cardiorespiratory fitness attenuate the adverse effects of severe/morbid obesity on cardiometabolic risk and insulin resistance in children? A pooled analysis. Diabetes Care $\mathbf{4 0}$, $1580-1587$

37. van Hees VT, Fang Z, Langford J, et al. (2014) Autocalibration of accelerometer data for free-living physical activity assessment using local gravity and temperature: an evaluation on four continents. I Appl Physiol 117, 738-744.

38. Migueles JH, Cadenas-Sanchez C, Ekelund U, et al. (2017) Accelerometer data collection and processing criteria to assess physical activity and other outcomes: a systematic review and practical considerations. Sport Med 47, 1821-1845.

39. Van Hees VT, Sabia S, Anderson KN, et al. (2015) A novel, open access method to assess sleep duration using a wristworn accelerometer. PLOS ONE 10, e0142533.

40. Hildebrand M, Van Hees VT, Hansen BH, et al. (2014) Age group comparability of raw accelerometer output from wristand hip-worn monitors. Med Sci Sports Exerc 46, 1816-1824.

41. van Ansem WJ, Schrijvers CT, Rodenburg G, et al. (2014) Maternal educational level and children's healthy eating behaviour: role of the home food environment (cross-sectional results from the INPACT study). Int J Behav Nutr Phys Act 11, 113.

42. Tanner JM \& Whitehouse RH (1976) Clinical longitudinal standards for height, weight, height velocity, weight velocity, and stages of puberty. Arch Dis Child 51, 170-179.

43. World Health Organization (2010) Global Recommendations on Physical Activity for Health. Geneva: WHO.

44. Prokasky A, Rudasill K, Molfese VJ, et al. (2017) Identifying child temperament types using cluster analysis in three samples. J Res Pers 67, 190-201.

45. Sanson A, Letcher P, Smart D, et al. (2009) Associations between early childhood temperament clusters and later psychosocial adjustment. Merrill Palmer Quart 55, 26-54.

46. Eshghi A, Haughton D, Legrand P, et al. (2011) Identifying groups: a comparison of methodology. J Data Sci $9,271-291$.

47. Johnson L, Wilks DC, Lindroos AK, et al. (2009) Reflections from a systematic review of dietary energy density and weight gain: is the inclusion of drinks valid? Obes Rev 10, 681-692.

48. Yin J, Xue H, Chen Y, et al. (2018) Dietary energy density is positively associated with body composition of adults in Southwest China. Public Health Nutr 21, 1827-1834.

49. DiMeglio DP \& Mattes RD (2000) Liquid versus solid carbohydrate: effects on food intake and body weight. Int J Obes Relat Metab Disord 24, 794-800.

50. Schröder H, Mendez MA, Gomez SF, et al. (2013) Energy density, diet quality, and central body fat in a nationwide survey of young Spaniards. Nutrition 29, 1350-1355.

51. Günther ALB, Stahl LJ, Buyken AE, et al. (2011) Association of dietary energy density in childhood with age and body fatness 
at the onset of the pubertal growth spurt. Br I Nutr $\mathbf{1 0 6}$ 345-349.

52. Johnson L, Mander AP, Jones LR, et al. (2008) A prospective analysis of dietary energy density at age 5 and 7 years and fatness at 9 years among UK children. Int $J$ Obes $\mathbf{3 2}$, 586-593.

53. Johnson L, van Jaarsveld CHM, Emmett PM, et al. (2009) Dietary energy density affects fat mass in early adolescence and is not modified by FTO variants. PLOS ONE $\mathbf{4}$, e4594.

54. Appannah G, Pot GK, Huang RC, et al. (2015) Identification of a dietary pattern associated with greater cardiometabolic risk in adolescence. Nutr Metab Cardiovasc Dis 25, 643-650.

55. Hallal PC, Andersen LB, Bull FC, et al. (2012) Global physical activity levels: surveillance progress, pitfalls, and prospects. Lancet 380, 247-257.

56. Hoffmann DA, Marx JM, Burmeister JM, et al. (2018) Friday night is pizza night: a comparison of children's dietary intake and maternal perceptions and feeding goals on weekdays and weekends. Int J Environ Res Public Health 15, 720.

57. Kerr A, Slater GJ \& Byrne N (2017) Impact of food and fluid intake on technical and biological measurement error in body composition assessment methods in athletes. Br J Nutr $\mathbf{1 1 7}$, 591-601.

58. Martinez-Vizcaino V, Martinez MS, Aguilar FS, et al. (2010) Validity of a single-factor model underlying the metabolic syndrome in children: a confirmatory factor analysis. Diabetes Care 33, 1370-1372.

59. Alberti KGMM, Eckel RH, Grundy SM, et al. (2009) Harmonizing the metabolic syndrome: a joint interim statement of the International Diabetes Federation Task Force on Epidemiology and Prevention; National Heart, Lung, and Blood Institute; American Heart Association; World Heart Federation; International Atherosclerosis Society; and International Association for the Study of Obesity. Circulation 120, 1640-1645. 